\title{
Restoration for Weakly Blurred and Strongly Noisy Images
}

\author{
Xiang Zhu and Peyman Milanfar \\ Electrical Engineering Department, University of California, Santa Cruz, CA 95064 \\ xzhudsoe.ucsc.edu, milanfardee.ucsc.edu
}

\begin{abstract}
In this paper we present an adaptive sharpening algorithm for restoration of an image which has been corrupted by mild blur, and strong noise. Most existing adaptive sharpening algorithms can not handle strong noise well due to the intrinsic contradiction between sharpening and denoising. To solve this problem we propose an algorithm that is capable of capturing local image structure and sharpness, and adjusting sharpening accordingly so that it effectively combines denoising and sharpening together without either noise magnification or over-sharpening artifacts. It also uses structure information from the luminance channel to remove artifacts in the chrominance channels. Experiments illustrate that compared with other sharpening approaches, our method can produce state of the art results under practical imaging conditions.
\end{abstract}

\section{Introduction}

Blur and noise are the two common problems that exist in digital imaging. An important camera setting that strongly affects these two distortions, and that needs to be carefully adjusted, is the aperture size. If the exposure time is fixed, a large aperture will increase the signal to noise ratio (SNR), meanwhile reducing the depth of field (DOF) and thus increasing the out-of-focus blur, which eliminates high-frequency components of the image. On the other hand, a small aperture will alleviate the blur but increase the noise level [17, 4]. Noise can also be suppressed by using longer exposure time; but of course, this may cause motion (either camera motion or object motion) blur that is even more difficult to remove $[3,14,1]$. At the same time, limited accuracy of auto-focus systems and low light condition may add extra blur and noise into the image. So in real applications, such as consumer digital imaging, it is very common to record weakly blurred and relatively noisy images (see Fig. 6 (a)).

In general, there are two possible types of techniques that can enhance the sharpness of an image under such conditions. One group of methods is blind-deconvolution. In recent years, several blind-deconvolution algorithms have been proposed to restore images degraded by blur $[3,13,5]$. These algorithms are generally designed under the assumption that the point spread function (PSF) of blur is spatially invariant, and that noise is very weak or virtually absent. Unfortunately, even when dealing with weakly blurred images, the presence of noise can be a significant problem for the state of the art deblurring algorithms. Consider the popular algorithms under the maximum a-posteriori (MAP) estimation framework, where total variation (TV) or other sparse image priors are frequently used [8, 13]. These regularization terms concentrate on smoothing pixels with median or small gradient values, leaving high-value gradients preserved. They perform as smoothing filter assuming a global model for the locally treated pixels without considering local image characteristics. Although they can provide a good balance between high frequency content restoration and noise suppression, the noise effect may still remain in the output data, and corrupt the smoothness of latent object structure. On the other hand, space invariance of the PSF does not hold in general for out-of-focus blur since the scene depth varies spatially [7]. Besides, high computational cost is another significant shortcoming of deblurring approaches [2].

Another group of techniques aiming at recovering weakly blurred images are the sharpening algorithms. The classic linear unsharp masking (UM) approach, which enhances certain high-frequency components of the input image, is popular in practice due to its simplicity. However, linear unsharp masking is very sensitive to noise and can easily produce overshoot artifacts [11]. Several adaptive versions of unsharp masking have been proposed to alleviate these problems [11, 12, 6, 18, 2]. In [11] the scaling factor that controls sharpening strength is determined according to local image characteristics measured by "image activity". In smooth and high-contrast areas, the scaling factors are reduced to avoid noise magnification or oversharpening artifacts. The problem for such algorithms is that they do not effectively suppress existing noise, while the image activity measure is also sensitive to local noise variation. So their performance is quite limited when the in- 
put noise is strong. The algorithm proposed in [2] incorporates a denoising filter to smooth the input image, and employs a clipping process to remove overshoot. However, this method smooths mostly low-frequency components of the input image, and enhances unfiltered high-frequency component directly, which again amplifies noise. Meanwhile the clipping process may affect structure smoothness generating artifacts especially for heavily noisy regions. Another sharpening algorithm called adaptive bilateral filter (ABF) can achieve good noise suppression [18]. ABF was designed by introducing an offset into the bilateral filter, which switches the behavior of the filter from denoising to edge sharpening according to local image structure. This method focuses on enhancing the the slope of edges, but its sharpening strength for texture or other image details is limited.

In this paper a new non-parametric approach called geometric locally adaptive sharpening (GLAS) is proposed for weakly blurred and strongly noisy images. The algorithm is derived from local adaptive regression [15]. The key idea behind this approach is sharpening according to the local image structure, so that denoising and sharpening processes can be effectively combined together. Overshoot artifacts are avoided by adjusting the local sharpening parameter according to a robust sharpness measure. Besides, the proposed method is also capable of removing structured color artifacts caused by, for example, the demosaicing process. The paper is organized as follows: In Section 2 we motivate and describe the proposed approach. Section 3 illustrates the restoration results for real images, and finally Section 4 provides the conclusion.

\section{Proposed algorithm}

In this section, we first propose an approach called geometric locally adaptive sharpening (GLAS). This approach takes local image structure into consideration, so that it can efficiently combine sharpening and denoising together. Then, the steering kernel (SK) regression technique for image reconstruction [15] is briefly introduced, which is able to capture local image structure even with presence of mild blur and strong noise. Based on the SK, a specific algorithm for constructing GLAS kernels for weakly blurred gray-scale image restoration is developed. Finally we extend this approach to color images with a strategy for removing chrominance artifacts.

\subsection{Motivation}

We model the process that degrades an ideal sharp image f (denoted in a lexicographically ordered vector) into the observed blurry and noisy data $\mathrm{g}$ as:

$$
\mathrm{g}=\mathbf{H f}+\mathbf{n}
$$

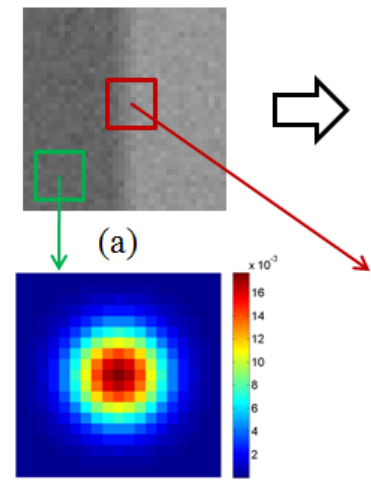

(c)

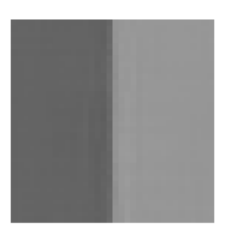

(b)

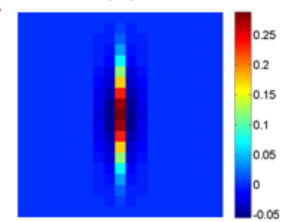

(d)
Figure 1. GLAS example. (a) blurred and noisy edge; (b) filtered edge; (c)-(d) Locally adaptive denoising/sharpening filters.

where $\mathbf{H}$ represents a blurring matrix, and $\mathbf{n}$ denotes additive noise. The fundamental problem of most sharpening methods is that they seek to find a global filter $\mathbf{S}$ that can do sharpening and denoising simultaneously:

$$
\hat{\mathbf{f}}=\mathbf{S g}=\mathbf{S H f}+\mathbf{S n}
$$

where the first term attempts to invert $\mathbf{H}$ globally : $\mathbf{S H} \approx \mathbf{I}$, and the second term unfortunately ends up amplifying the noise. One example is the classic linear $\mathbf{U M}$, whose $\mathbf{S}$ can be represented as:

$$
\mathbf{S}=\mathbf{I}+\lambda \mathbf{L}
$$

where $\mathbf{I}$ is the identity, and $\mathbf{L}$ denotes a Laplacian (highpass) filter matrix. We can see that Sn preserves all the noise, meanwhile amplifying its high-frequency components by the factor $1+\lambda$.

One possible way to avoid noise magnification is to design a sharpening filter adaptive to the local image statistics. In this alternative view, it is not necessary to globally invert the blur operator $\mathbf{H}$. Instead, its action should be locally controlled to respond to the signal characteristics implied in the measured image. That is to say, for instance, in places where the effect of blur is not felt (such as flat areas of the image) the sharpening filter should concentrate more on noise reduction. Meanwhile, in edge-containing regions, the filter should attempt to sharpen the image only in the edge profile direction, and still effect smoothing along the edge direction. (See example shown in Fig. 1)

We term this approach geometric locally adaptive sharpening (GLAS). In order to implement GLAS, a technique that can capture the local image structure even in the presence of blur and noise is required first. Many sophisticated adaptive denoising filters (e.g. Bilateral filter [16], NLM [9], steering kernel regression [15], etc.) can achieve this task. Here we chose steering kernel (SK) as a starting point, which is exceedingly robust to noise and perturbations of local image data. 


\subsection{Steering kernel construction}

The key idea behind SK is to robustly obtain the local structure of images by analyzing estimated gradients, and use this structure information to determine the shape and size of a canonical kernel [15]. In earlier work, SK has been successfully utilized to address the image denoising problem.

Assuming a pixel of interest is located at position $\mathbf{x}_{i}=$ $\left[x_{i}, y_{i}\right]$, its $\mathrm{SK}$ is mathematically represented as:

$K\left(\mathbf{x}_{l}-\mathbf{x}_{i}\right)=\sqrt{\operatorname{det}\left(\mathbf{C}_{l}\right)} \exp \left\{-\left(\mathbf{x}_{l}-\mathbf{x}_{i}\right)^{T} \mathbf{C}_{l}\left(\mathbf{x}_{l}-\mathbf{x}_{i}\right)\right\}$

where $\mathbf{x}_{l}$ denotes a given location inside the SK window centered at $\mathbf{x}_{i}$, and $\mathbf{C}_{l}$ is a covariance matrix estimated from a collection of gradients within an analysis window $w_{l}$ around $\mathbf{x}_{l}$ (more on this below).

Examples of the SK in a weakly blurred image (added with white Gaussian noise) are given in Fig. 2 (a)-(c), where we can see that the estimated kernels reliably capture local image structures. Since the definition (4) of the SK seems like a simple Gaussian function, it may appear mysterious that the resulting kernel values do not simply have elliptical contours. This is a subtle, but important point, which stems from the fact that a separate covariance matrix $\mathbf{C}_{l}$ is estimated and used at each pixel location. This yields a far richer set of shapes for the resulting kernel weights, as seen in Fig. 2, than would otherwise be expected of the humble Gaussian kernel. In the flat region, the SK is wide and basically isotropic, indicating that there is no strong directional structure inside this area. In the edge region, the shape of SK depicts the edge outline, and the kernel values basically indicate the pixel intensity similarity with respect to the pixel of interest. In the region that contains small scale image details, the corresponding SK also shrinks to a small region. Besides, it can be observed that the SKs are basically robust to high levels of noise.

Let us describe more precisely how the kernel values in (4) are computed. The local gradient matrix for the window $w_{l}$ centered at $\mathbf{x}_{l}$ is defined as:

$$
\mathbf{D}=\left[\begin{array}{cc}
\vdots & \vdots \\
G_{x}\left(\mathbf{x}_{m}\right) & G_{y}\left(\mathbf{x}_{m}\right) \\
\vdots & \vdots
\end{array}\right], \quad \mathbf{x}_{m} \in w_{l}
$$

where $\left[G_{x}\left(\mathbf{x}_{m}\right), G_{y}\left(\mathbf{x}_{m}\right)\right]$ denote the image gradient at $\left[x_{m}, y_{m}\right]$. The dominant direction $\mathbf{v}_{1}$ and its perpendicular direction $\mathbf{v}_{2}$ within the region $w_{l}$ can be estimated by computing the (compact) singular value decomposition (SVD) of D:

$$
\mathbf{D}=\mathbf{U} \boldsymbol{\Lambda} \mathbf{V}^{T}=\mathbf{U}\left[\begin{array}{cc}
s_{1} & 0 \\
0 & s_{2}
\end{array}\right]\left[\begin{array}{ll}
\mathbf{v}_{1} & \mathbf{v}_{2}
\end{array}\right]^{T}
$$

Here the singular values $s_{1} \geq s_{2} \geq 0$ represent the energy in the directions $\mathbf{v}_{1}$ and $\mathbf{v}_{2}$ respectively. The matrix $\mathbf{C}_{l}$

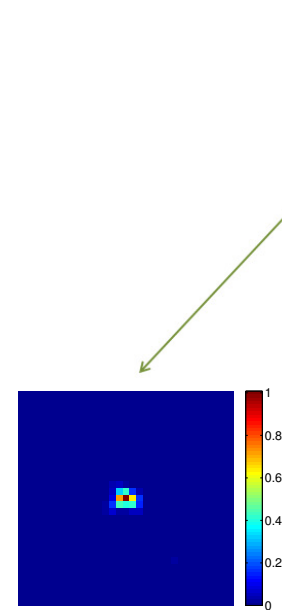

(a)

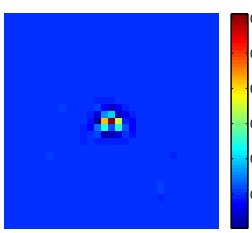

(d)

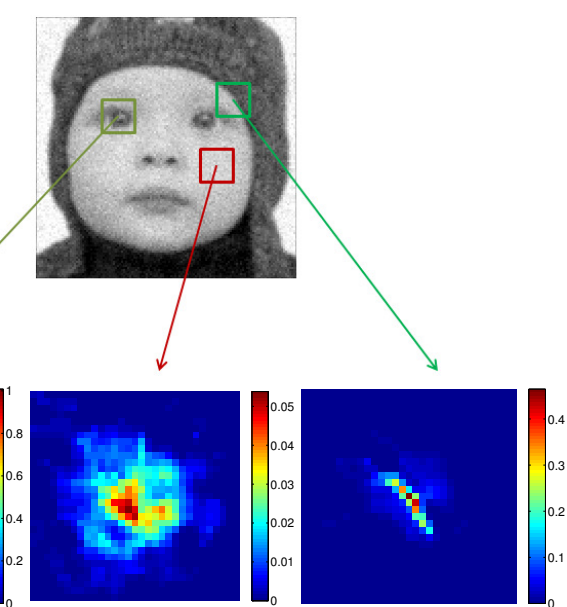

(b)

(c)

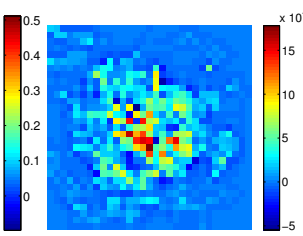

(e)

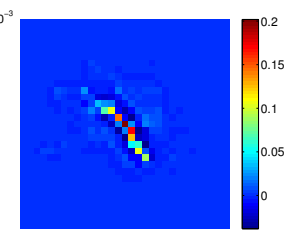

(f)
Figure 2. Examples of SK (top row) and GLAS (bottom row) kernels in selected regions: (a)-(c): steering kernels; (d)-(f): GLAS kernels.

can then be stably estimated through the following formula [15]:

$$
\mathbf{C}_{l}=\gamma\left(\varrho_{1} \mathbf{v}_{1} \mathbf{v}_{1}^{T}+\varrho_{2} \mathbf{v}_{2} \mathbf{v}_{2}^{T}\right)
$$

with

$$
\begin{gathered}
\varrho_{1}=\frac{s_{1}+\lambda^{\prime}}{s_{2}+\lambda^{\prime}}, \quad \varrho_{2}=\frac{1}{\varrho_{1}} \\
\gamma=\left(\frac{s_{1} s_{2}+\lambda^{\prime \prime}}{M}\right)^{\alpha}
\end{gathered}
$$

where $\varrho_{1}$ and $\gamma$ are the elongation and scaling parameters, respectively. $\lambda^{\prime}$ and $\lambda^{\prime \prime}$ are regularization parameters that dampen the noise effect and restrict $\varrho_{q}$ and $\gamma$ from becoming zero. $M$ is the number of samples within the analysis window $w_{l}$. The scalar $\alpha$ controls how strongly the size of the kernel will be affected by the local structure. ${ }^{1}$

\subsection{The GLAS kernel}

Once the steering kernel $K$ is obtained, we build the geometric locally adaptive sharpening (GLAS) kernel $S$ through the formula:

$$
S=K+q L \otimes K
$$

\footnotetext{
${ }^{1}$ We fix $\lambda^{\prime}=\lambda^{\prime \prime}=0.0001$ through out the paper. The structure sensitivity $0<\alpha<1.5$ should be set according to the noise condition of the image. Generally, the stronger the noise is, the lower the value of $\alpha$ should become.
} 
where $\otimes$ represents the convolution operator, and $L$ denotes a Laplacian filter. The positive scalar $q$ determines the degree of sharpening (more on this below). The restored image is then computed as a local regression (weighted average) with the kernel $S$ as follows:

$$
\hat{f}\left(\mathbf{x}_{i}\right)=\frac{\sum_{\mathbf{x} \in w_{l}} S\left(\mathbf{x}-\mathbf{x}_{i}\right) g(\mathbf{x})}{\sum_{\mathbf{x} \in w_{l}} S\left(\mathbf{x}-\mathbf{x}_{i}\right)}
$$

where $g$ is the measured blurry and noisy image. Fig. 2 (d)(f) illustrate examples of GLAS kernels. Consider the edge pixel for instance. The negative values appearing along the two sides of the edge outline indicate that the sharpening effect happens in the direction perpendicular to the edge orientation. Meanwhile, we have most positive values along the outline of the edge, which means the kernel smooths (denoises) the edge simultaneously. On the other hand, in the flat region, though there exist few small negative values across the region, the kernel can still be viewed largely as a smoothing filter.

One problem that frequently arises in practice, in part due to limited depth of field of cameras, is that the degree of blur varies in space. As a result, with a globally designed sharpening approach, some regions that happen to be already in-focus may be over-sharpened, resulting in overshoots or ringing artifacts. Without additional finesse, similar problems could plague the proposed method. If the local region is already in focus, a fixed, and unnecessarily high value of $q$ would produce overshoots along the edge pixels. To alleviate this problem, we next make the sharpening parameter $q$ adaptive to the sharpness of local image content.

An image sharpness metric, which can estimate the local image blur even in the presence of noise [20, 19], is implemented here. The metric is based on the singular values of local gradient matrix, which have already been calculated in the SK construction stage, so it does not require much extra calculation. The local metric $Q$ for the pixel located at $\mathbf{x}_{l}$ is defined as [20, 19]:

$$
Q=s_{1} \frac{s_{1}-s_{2}}{s_{1}+s_{2}}
$$

where $s_{1}, s_{2}$ were introduced in (6). The blurrier the local patch of the image is, the lower the $Q$ value becomes.

To avoid overshoots in already sharp edges, we set the values of the parameter $q$ for a given pixel as a function of the local value of $Q$ as follows (see Fig. 3):

$$
q= \begin{cases}\beta & \text { if } \quad Q<\tau_{1} \\ \beta\left(Q-\tau_{2}\right) /\left(\tau_{1}-\tau_{2}\right) & \text { if } \quad \tau_{2}>Q \geq \tau_{1} \\ 0 & \text { if } Q \geq \tau_{2}\end{cases}
$$

This formulation penalizes $q$ in regions with high value of ${ }^{2}$ Q.

\footnotetext{
${ }^{2}$ For all the experiments shown in this paper, the analysis window $w_{l}$
}

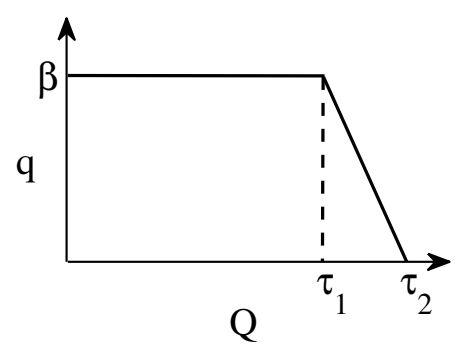

Figure 3. Enhancement Function defined in (12).

The proposed sharpening process (Equation (9)-(12)) can also be described in the following vector form:

$$
\begin{aligned}
\hat{\mathbf{f}} & =\mathbf{D}_{S}^{-1} \mathbf{S g} \\
& =\mathbf{D}_{S}^{-1}(\mathbf{K}+\mathbf{Q K L}) \mathbf{g}
\end{aligned}
$$

Here $\mathbf{S}$ is the GLAS sharpening matrix, $\mathbf{K}$ is the steering kernel matrix, whose entry $K_{i, j}=K\left(\mathbf{x}_{j}-\mathbf{x}_{i}\right)$ as defined in (4), and $\mathbf{D}_{S}$ is the diagonal matrix of row sums of $\mathbf{S}$ which act as normalization factors. $\mathbf{Q}=\operatorname{diag}\left(q_{1}, q_{2}, \ldots\right)$ assigns to each pixel the corresponding sharpening parameter.

\subsection{Chrominance artifact removal}

Digital color images are often corrupted by not only luminance noise but also chrominance artifacts during imaging. These artifacts are not pure white noise [10], and may contain some visually annoying structures. For example, the false color artifacts caused by the demosaicing process look like small colored bars, which may appear across edges of an object. Besides, since the overall contrast of chrominance channels is usually weak in natural images, the effect of chrominance artifacts may be worsened by JPEG compression when the contrast of artifacts appear stronger than that of real objects (see Fig. 4 (a), (b)). Under such conditions, it is very difficult to remove artifacts while restoring object structure by denoising chrominance channels independently. Therefore, the object structure information from the luminance channel should be utilized to effect chrominance channel restoration. This idea is easily implemented using the proposed GLAS kernel.

As described Section 2.2, SK is capable of reliably capturing the object structures from a gray-scale image. These estimated kernels can, of course, be used for chrominance channels as well. We observe that restored chrominance channels do not need to be as sharp as the luminance channel, since the human visual system is less sensitive to subtle color variations. So the GLAS kernels for chrominance channels can be produced using a small constant $q$ (e.g. $q=0.1$ ) to suppress artifacts effectively. A representative

is set to size $5 \times 5$, and $\tau_{1}=150, \tau_{2}=200$. We set the scalar $\beta=1.5$, and it can also be tuned according to the level of sharpening required by the user. 
example is given in Fig. 4, where it can be seen that the proposed strategy successfully suppressed basically all the artifacts (including structured artifacts), meanwhile reconstructing high-frequency content rather well in chrominance channels.

To summarize, the overall algorithm can be described as follows:

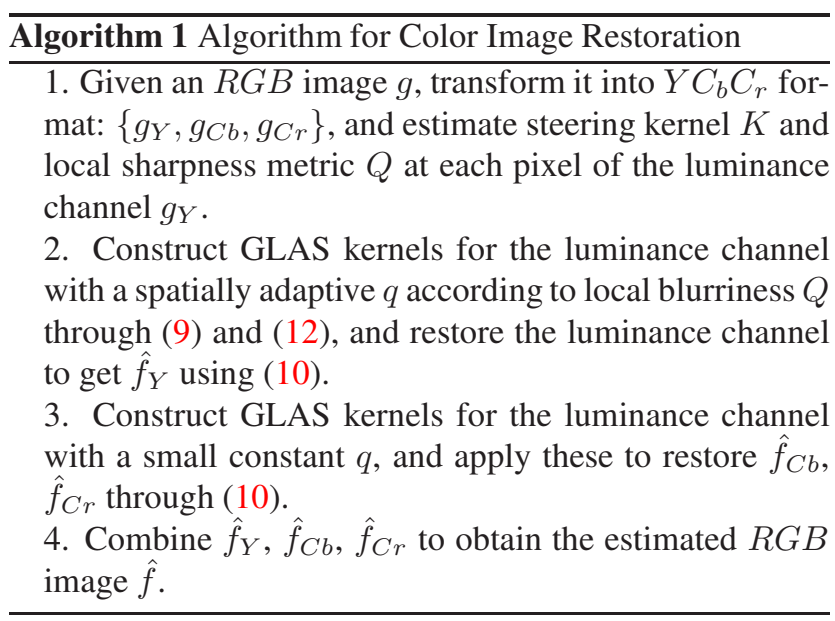

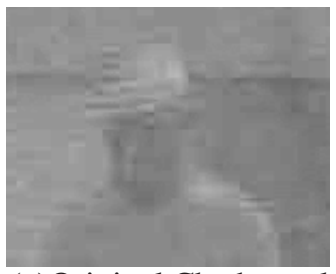

(a)Original Cb channel

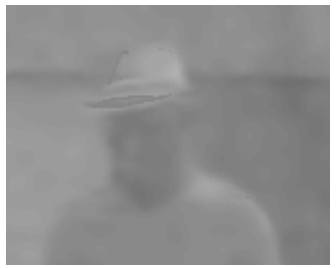

(c)Restored $\mathrm{Cb}$ channel

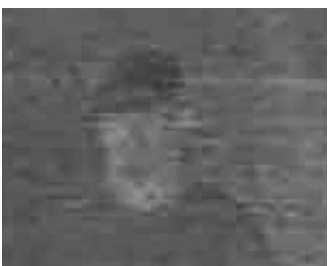

(b)Original $\mathrm{Cr}$ channel

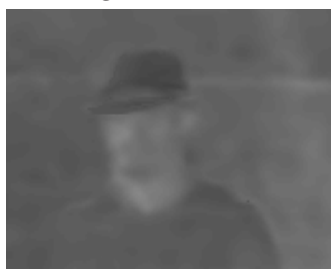

(d)Restored Cr channel
Figure 4. Examples of chrominance channel restoration using our proposed method.

\section{Experimental results}

To show the effectiveness of the proposed algorithm, we test it on several real images that suffer from mild blur and strong noise. Several leading adaptive sharpening approaches (Adaptive UM [11], Constrained UM [2] and ABF [18]) are also applied as comparison.

One set of results are given in Fig. 5, where (a) illustrates an image taken by a Canon Rebel T1i camera at ISO3200, which contains strong shot noise and mild outof-focus blur. In (b) the Adaptive UM produced result with sharpened edges and detail, but the noise is also strongly amplified. ${ }^{3}$ A similar situation happens in Constrained UM in (c). Although it did not significantly raise the noise lever, the high-frequency noise artifacts can be easily observed especially in the flat areas. In (d), ABF provides an image with the noise sufficiently suppressed, since it incorporates bilateral filter. It sharpens edges well, but its sharpening effect in detail regions is limited. In (e), our proposed algorithm suppressed both blur and noise. The surface of the doll and the background are very clean, while the restored details are at least as good as (b) and (c).

The next set of experimental results are shown in Fig. 6. The original image in (a) suffers from mild out-offocus blur and looks noisy because of bad illumination and JPEG compression. Constrained UM restored image in (b) looks much sharper, but the noise effects are also magnified, which is probably because in the Constrained UM algorithm unfiltered high-frequency components of the input image are directly added to the output. ABF successfully removed random noise and blocking artifacts caused by compression, but again the sharpening strength is not strong. Although it makes edges sharper without any overshoot, some image details are smoothed. So visually the result in (c) looks more blurry compared with (b) or (d). The proposed method successfully removed both blur and noise artifacts, making the restored structure more natural (see Fig. 6 (d)). It also suppressed false color.

Additional results are given in Fig. 7, where again Constrained UM and ABF do not perform well in either noise suppression or sharpening. On the other hand, this is a typical example of strong chrominance artifacts, which are effectively removed only by the proposed algorithm. (see Fig. 7 (b)-(d)).

\section{Conclusion}

In this paper, we developed a locally adaptive restoration algorithm based on an extension of the steering kernel regression technique. This algorithm is able to capture local image structure and thus effectively combine denoising and sharpening together. Experiments show that the proposed approach can efficiently restore images distorted by weak blur and strong noise. Compared with other state of the art adaptive sharpening methods, it handles both denoising and sharpening tasks simultaneously very well, and is capable of removing chrominance artifacts. This algorithm is also computationally cheap, since it is not iterative.

\section{References}

[1] L. Bar, B. Berkels, G. Sapiro, and M. Rumpf. A variational framework for simultaneous motion estimation and restora-

\footnotetext{
${ }^{3}$ In general, Adaptive UM [11] produces similar sharpening effects as Constrained UM [2], but with stronger noise artifacts. Its experimental results for the remaining test images are not given due to space limit.
} 

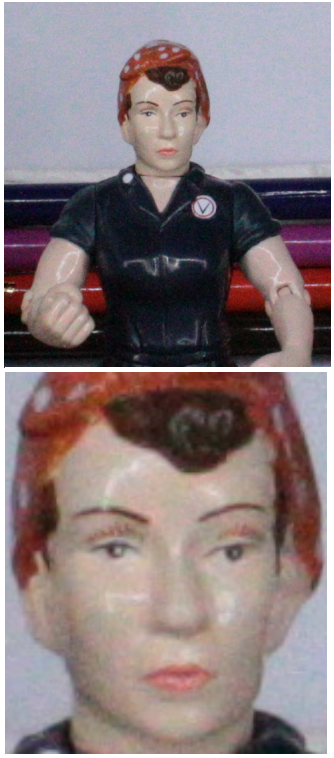

(a) Original image
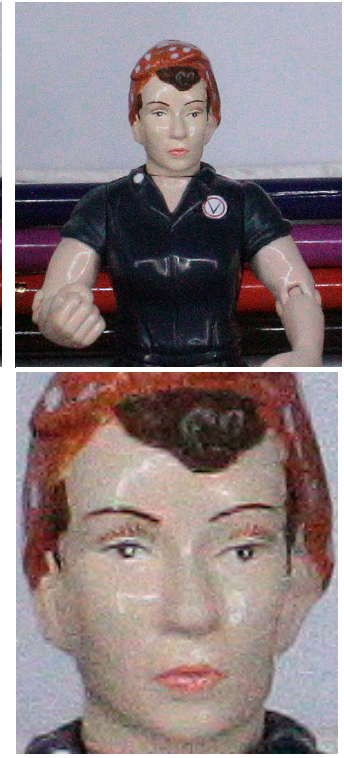

(b) Adaptive UM
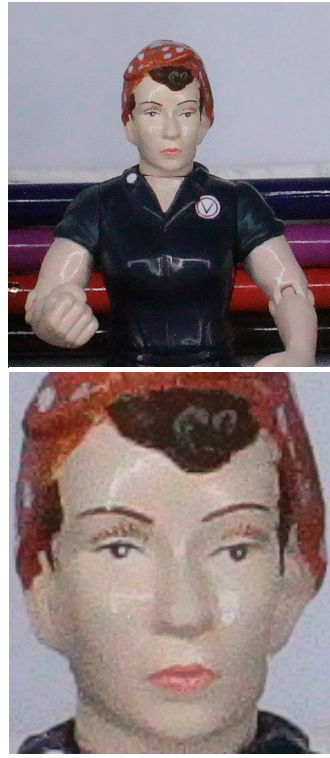

(c) Constrained UM
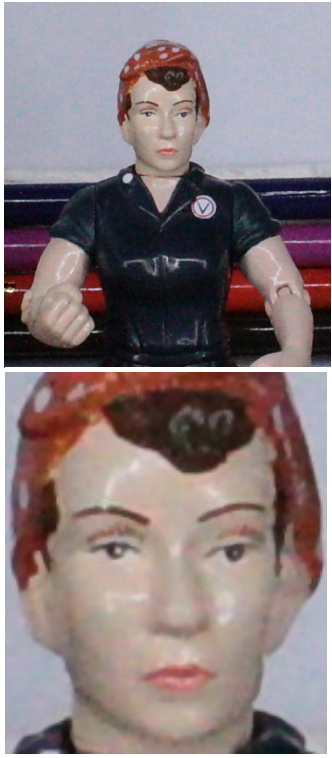

(d) $\mathrm{ABF}$
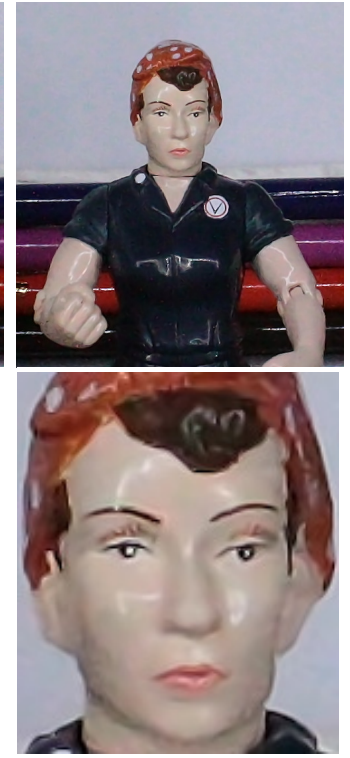

(e) Proposed method

Figure 5. Experimental results: (a) input image; (b) result of Adaptive UM [11]; (c) result of Constrained UM [2]; (d) result of ABF [18]; (e) result of Proposed method.

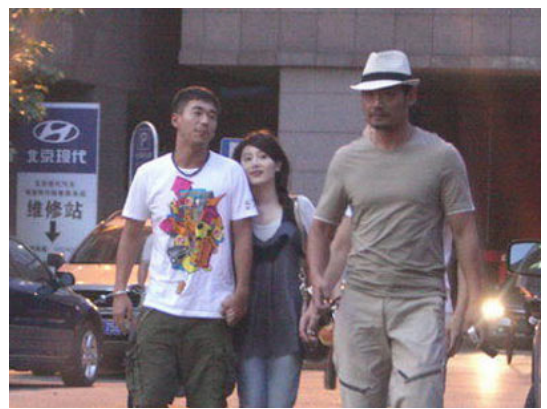

(a) Original image

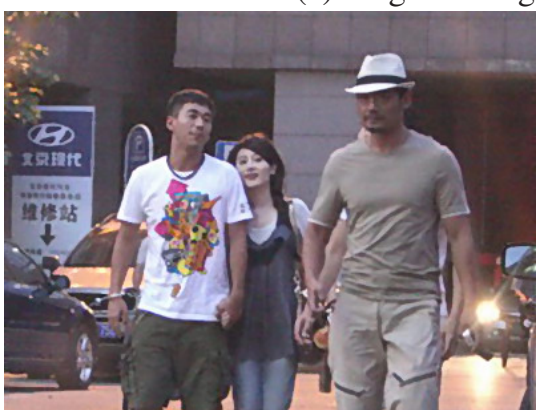

(c) $\mathrm{ABF}$
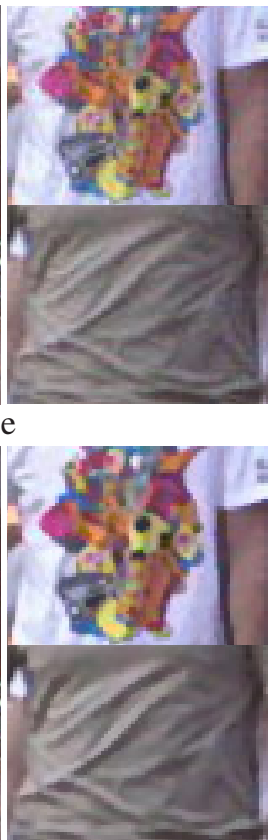
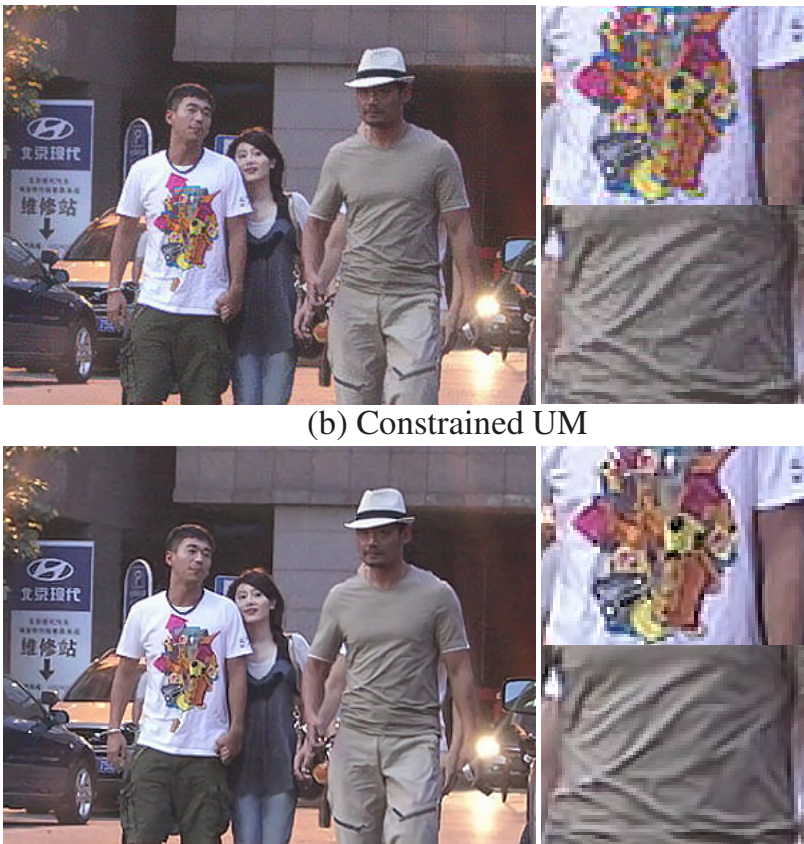

(b) Constrained UM

(d) The proposed method

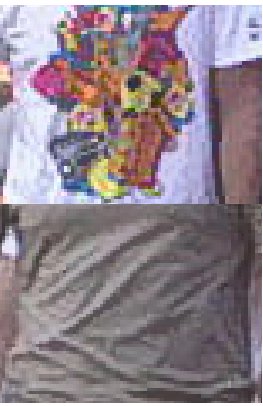

Figure 6. Experimental results of sharpening a weakly blurred and strongly noisy image. (a) input image; (b) result of Constrained UM [2]; (c) result of ABF [18]; (d) result of Proposed method.

tion of motion-blurred video. Proc. International Conference on Computer Vision (ICCV07), October 2007. 1

[2] R. C. Bilcu and M. Vehvilainen. Constrained unsharp masking for image enhancement. Proc. 3rd International Conference on Image and Signal Processing, pages 10-19, 2008. 1, $2,5,6,7$

[3] R. Fergus, B. Singh, A. Hertsmann, S. T. Roweis, and W. T.
Freeman. Removing camera shake from a single image. ACM Transactions on Graphics (SIGGRAPH), 2006. 1

[4] J. Jia, J. Sun, C. Tang, and H. Shum. Bayesian correction of image intensity with spatial consideration. $E C C V$, pages 342-354, 2004. 1

[5] N. Joshi, R. Szeliski, and D. Kriegman. PSF estimation using sharp edge prediction. CVPR, 2008. 1 


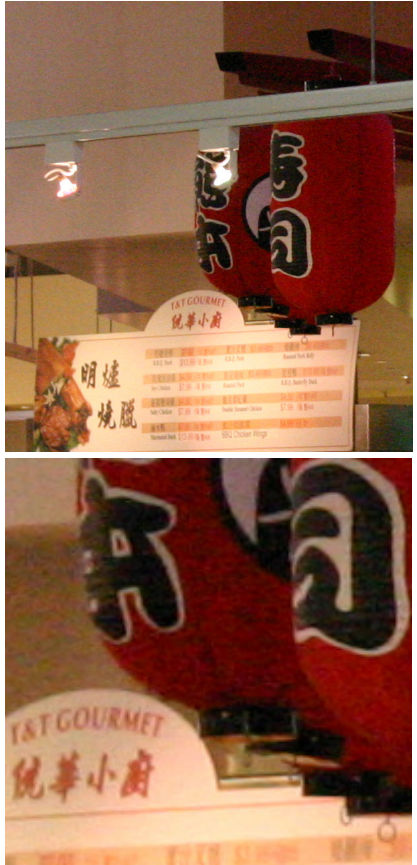

(a) Original image

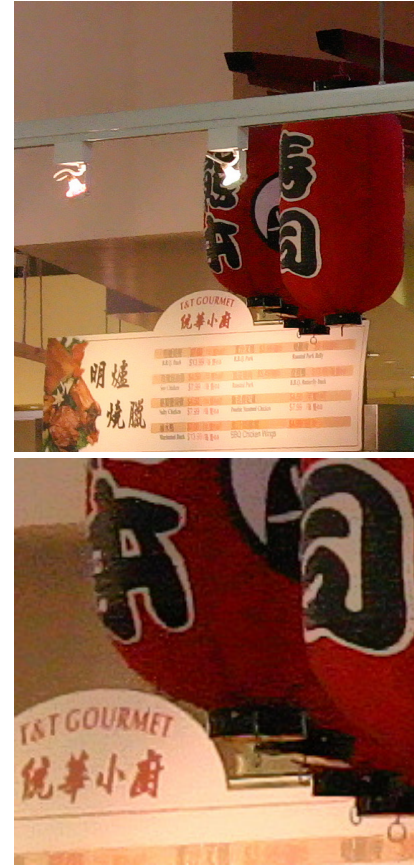

(b) Constrained UM

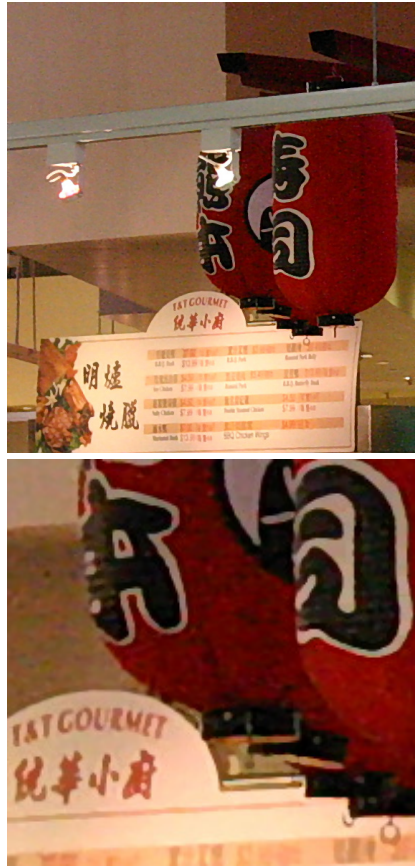

(c) $\mathrm{ABF}$

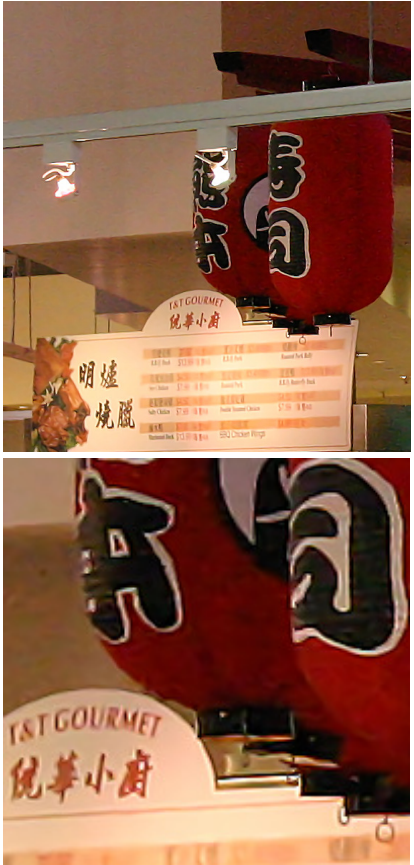

(d) Proposed method

Figure 7. Experimental results: (a) input image; (b) result of Constrained UM [2]; (c) result of ABF [18]; (d) result of Proposed method.

[6] S. Kim and J. P. Allebach. Optimal unsharp mask for image sharpening and noise removal. J. Electron. Imag., 14(2):023007-1, 2005. 1

[7] A. Levin, R. Fergus, F. Durand, and W. T. Freeman. Image and depth from a conventional camera with a coded aperture. ACM Transactions on Graphics (SIGGRAPH), 2007. 1

[8] A. Levin, Y. Weiss, F. Durand, and W. T. Freeman. Understanding and evaluating blind deconvolution algorithms. CVPR, 2009. 1

[9] M. Mahmoudi and G. Sapiro. Fast image and video denoising via nonlocal means of similar neighborhoods. IEEE Signal Processing Letters, 12(12):839-842, Dec. 2005. 2

[10] S. H. Park, H. S. Kim, S. Lansel, M. Parmar, and B. Wandell. A case for denoising before demosaicking. In Asilomar Conference on Signals, Systems, and Computers, Pacific Grove, CA, Nov. 2009. 4

[11] A. Polesel, G. Ramponi, and V. J. Mathews. Image enhancement via adaptive unsharp masking. IEEE Transactions on Image Processing, 9(3):505-510, March 2000. 1, 5, 6

[12] F. Russo. An image-enhancement system based on noise estimation. IEEE Trans. Instrumentation and Measurement, 56(4):1435-1442, August 2007. 1

[13] Q. Shan, J. Jia, and A. Agarwala. High-quality motion deblurring from a single image. ACM Transactions on Graphics (SIGGRAPH), 2008. 1

[14] M. Sorel and P. Sroubek. Space-variant deblurring using one blurred and one underexposed image. Proceedings of the 16th IEEE International Conference on Image Processing, pages 157-160, 2009. 1
[15] H. Takeda, S. Farsiu, and P. Milanfar. Kernel regression for image processing and reconstruction. IEEE Transactions on Image Processing, 16(2):349-366, February 2007. 2, 3

[16] C. Tomasi and R. Manduchi. Bilateral filtering for gray and color images. Proceeding of the 1998 IEEE International Conference of Compute Vision, Bombay, India, pages 836846, January 1998. 2

[17] L. Yuan, J. Sun, L. Quan, and H. Shum. Image deblurring with blurred/noisy image pairs. ACM Transactions on Graphics (SIGGRAPH), 2007. 1

[18] B. Zhang and J. P. Allebach. Adaptive bilateral filter for sharpness enhancement and noise removal. IEEE Transactions on Image Processing, 17(5):664-678, May 2008. 1, 2, $5,6,7$

[19] X. Zhu and P. Milanfar. Automatic parameter selection for denoising algorithms using a no-reference measure of image content. Accepted for IEEE Transactions on Image Processing: 10.1109/TIP.2010.2052820. ISSN: 1057-7149. 4

[20] X. Zhu and P. Milanfar. A no-reference sharpness metric sensitive to blur and noise. International Workshop on Quality of Multimedia Experience (QoMEX), 2009. 4 